\title{
Clinical Trial Branch
}

National Cancer Institute

\section{Source}

National Cancer Institute. Clinical Trial Branch. NCI Thesaurus. Code C82497.

A subject categorization in clinical trials based on the conditions a subject has previously met. 